\title{
Burns in Tehran: demographic, etiological, and clinical trends
}

\author{
Mohammad Ali Bahar ${ }^{\mathrm{a}}$, Mohammadreza Pakyari ${ }^{\mathrm{b}}$, Rayeheh Bahar ${ }^{\mathrm{b}}$ \\ ${ }^{a}$ Burn Research Center, Iran University of Medical Sciences and Health Services, Tehran 14155-655, \\ ${ }^{b}$ Shiraz University of Medical Sciences, Shiraz 71345-1583, Iran
}

\begin{abstract}
Background: Burns are a major public health problem. They often require intensive care and long periods of hospitalization. In Tehran, about 5\% of all hospitalized injuries are burns. There are no published long-term epidemiological studies regarding burn injuries of adults in Iran.

Objective: To identify risk factors for burn injuries and provide a starting point for the establishment of an effective prevention plan.

Methods: We analyzed the demographic, etiological, and clinical data of 1860 burn patients admitted to a major acute care hospital in Tehran between March 2010 and April 2011. Data were obtained from the registry recorded in Shahid Motahari Trauma Hospital and evaluated using a chi-square test.

Results: Males were more than twice as likely to be burn patients than females (72.0\% vs. 28.0\%). Second and third-degree burns with a body surface area of 21\%-30\% constituted the highest injury reported (75.3\%). The most common causes of the recorded burns were natural gas, gasoline (42\%) and open fire (10.2\%). Unintentional burns were reported in $85 \%$ of the cases, and $15 \%$ of the burn victims were suicide-related incidents; mainly among women. In $75 \%$ of suicide attempts, women set themselves on fire to commit suicide. The mean duration of hospitalization was 25 days and the mortality rate was $10.7 \%$. Mean age of reported deaths was 38.6 years; with a mean of 30 years among women and 51.5 years among men.

Conclusion: The group at highest risk was young men 21-30 years old. However, an astonishing finding was that $75 \%$ of suicidal-related incidents involved women setting themselves on fire. Those with the highest mortality rate were victims of burns with gas, gasoline, and kerosene; with a mean age of 30 years of death among women.
\end{abstract}

Keywords: Burns, demographic data, etiology, hospitalization, Iran, morbidity, mortality, Tehran

Burn injuries represent a significant public health concern worldwide because of the increased length of stay in intensive care units and general wards, nosocomial infections, as well as their high cost [1-3]. In Iran, burn-related injuries constitute about $18 \%$ of all hospitalized trauma cases in children and $5 \%$ in adults [4]. In Tehran, Shahid Motahari Hospital has a specialized burn unit that is considered a tertiary referral center. However, because of geographical proximity and being the only extensive care unit, most burn patients arrive directly to the center and are not referred from other small hospitals, thus representing the majority of burn patients with various etiologies in Tehran.

A large percentage of burns occur accidentally. This could be reduced by effective prevention plans

Correspondence to: Dr. Mohammad Ali Bahar, Burn Research Center, Iran University of Medical Sciences and Health Services, Tehran 14155-6559, Iran. E-mail: mobahar@yahoo.com based on the identification of major risk groups (of sex and age) and on safety pitfalls [1]. In Iran, these data have been systematically collected and analyzed, but plans and follow-up did not reach the proposed goals. Therefore, data between March 2010 and April 2011 were re-extracted in an effort to identify risk groups and devise a new prevention plan. This study presents pertinent demographic, etiological, and clinical data essential for establishing such a plan.

\section{Methods}

We analyzed data from 1860 consecutive burn patients admitted for at least one day to the Shahid Motahari Burn Referral Center. Data were obtained from the hospital registry. This study and access to the data was approved after being considered by our local medical ethics committee. We collected demographic and clinical data using a form on all participants by completed by dedicated research 
personnel. These include the nature and mechanism of trauma, degree indices, in-hospital evaluation and initial treatment, medical and surgical management, and procedures performed. The demographic data (e.g., age and sex), etiological factors, clinical characteristics (e.g., severity of burn, type of injury, and treatment), and the outcome were analyzed and presented in this study. The total body surface area (TBSA) percentage index was used to define burn severity.

\section{Results}

During the period surveyed (2010-2011), 1860 burn patients were admitted and hospitalized for at least one day at Shahid Motahari, the only trauma center for burns in Tehran.

\section{Demographic characteristics}

Sex: Between March 2010 and April 2011 the distribution of burns according to sex remained almost constant with a clear male predominance. The mean prevalence of male burn patients was $72 \%$. From all the recorded hospitalizations, $15 \%$ were suicidalrelated incidents. In addition, $75 \%$ of hospitalized women who attempted to commit suicide did so by setting themselves on fire.

Age: Throughout the study period, young adults 2130 years of age comprised the largest age group, with a prevalence of $33.9 \%$ of cases. Children less than 10 years old comprised $12.4 \%$ of burn patients
(Figure 1).

Etiology: Explosives-related injury was uncommon $(0.5 \%)$, while natural gas and gasoline accounted for $42 \%$ of burns. An open flame was the second most common cause with a mean incidence of $10.2 \%$, and kerosene was the third most frequent at $9.7 \%$ (Figure 2).

Clinical characteristics: Second and third degree burns accounted for more than three quarters of hospitalizations in the study period (75.3\%). Burns comprising the upper body and trunk were the highest reported injury with an prevalence of $22.6 \%$.

Hospitalization, procedures, and mortality: The mean duration of hospitalization was 25 days. An overwhelming portion of patients $(21.5 \%)$ were admitted in the last two months of the Iranian calendar year (February and March). Surgical procedures became more common over the reviewed months: the lowest percentage was in March 2010 (13.4\%) and the highest in February 2011 (26.6\%). The mortality rate was $10.7 \%$. The mean age of patients at death was 38.6 years, with 30 years in female patients and 51.5 years in male patients (Table 1). The primary cause of death was septicemia (63.21\%) followed by acute renal failure (19.36\%) (Table 2). Overall, 64\% of patients were discharged from the hospital upon the completion of their treatments, while around $25.3 \%$ of the patients left the hospital before the end of treatment period either to complete it as outpatients or against advice.

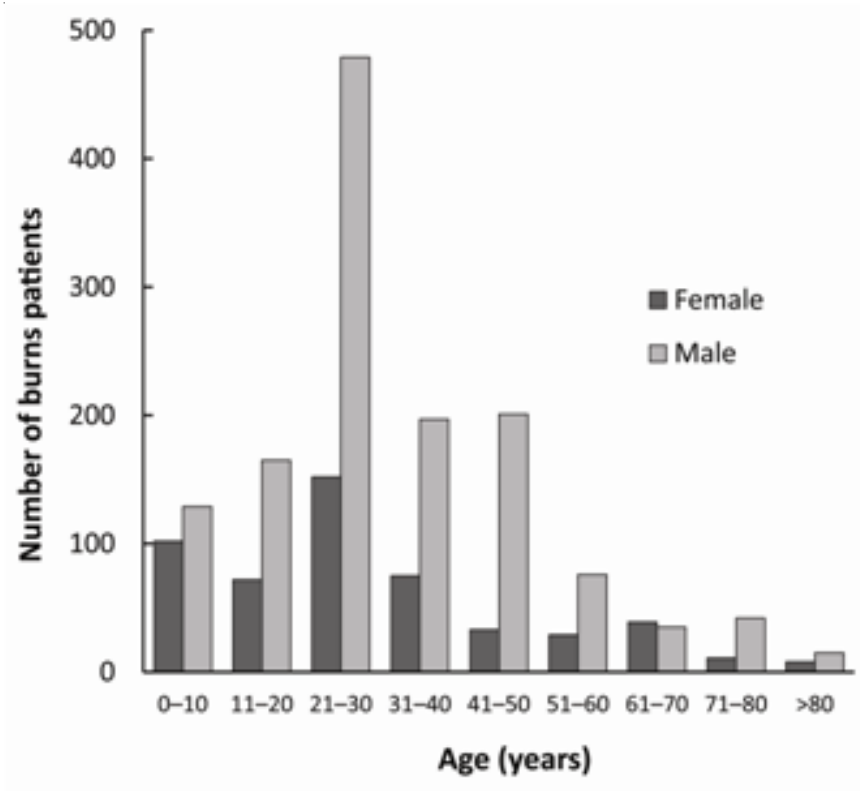

Figure 1. Age distribution of patients 


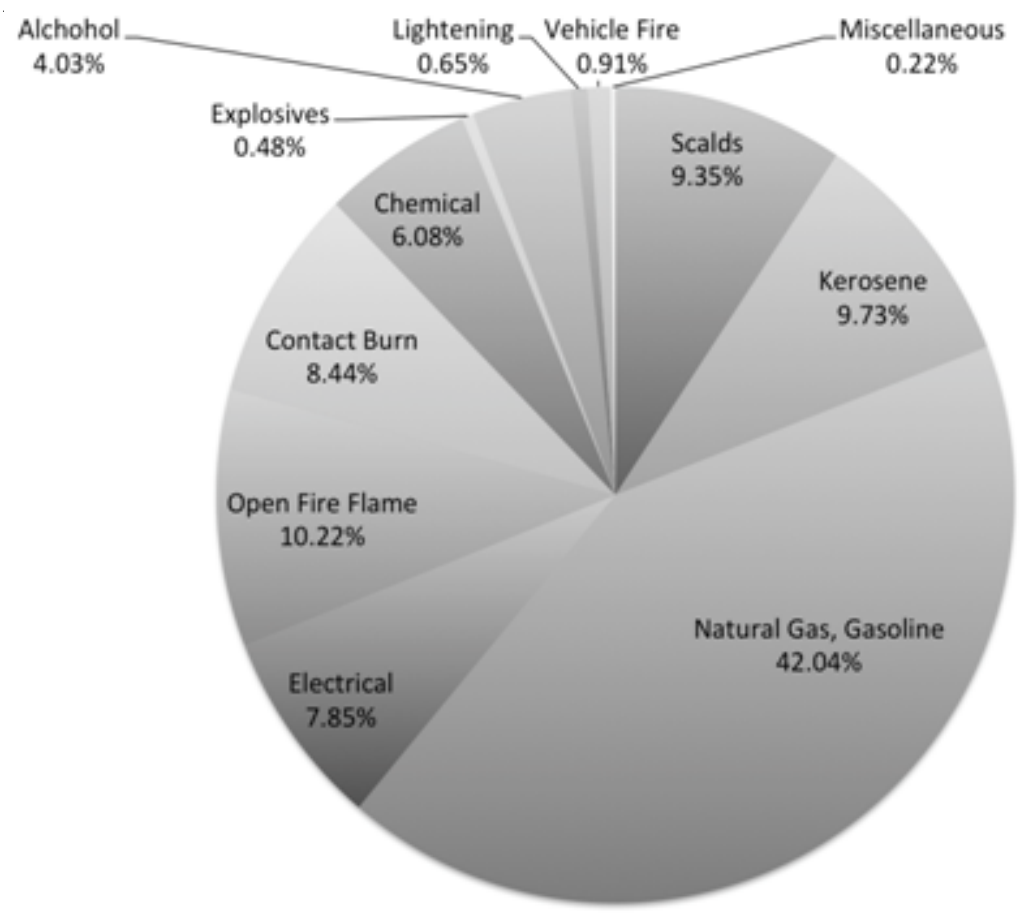

Figure 2. Causes of burns for patients at Motahari Referral Burn Center, Tehran, Iran

Table 1. Mortality rates by age groups

\begin{tabular}{lccc}
\hline Age (year) & Female & Male & Percentage \\
\hline $0-10$ & 5 & 8 & 13 \\
$11-20$ & 8 & 12 & 20 \\
$21-30$ & 26 & 16 & 42 \\
$31-40$ & 22 & 25 & 47 \\
$41-50$ & 9 & 34 & 43 \\
$51-60$ & 3 & 15 & 18 \\
$61-70$ & 2 & 4 & 6 \\
$71-80$ & 0 & 5 & 5 \\
$>80$ & 2 & 3 & 5 \\
Total & 77 & 122 & 199 \\
\hline
\end{tabular}

Table 2. Causes of mortality

\begin{tabular}{llll}
\hline & Female & Male & Total \\
\hline Septicemia & $48(24 \%)$ & $78(39 \%)$ & $126(63 \%)$ \\
Acute renal failure & $15(8 \%)$ & $24(12 \%)$ & $39(19 \%)$ \\
Shock & $12(6 \%)$ & $20(10 \%)$ & $32(16 \%)$ \\
Respiratory failure & $2(1 \%)$ & - & $2(1 \%)$ \\
Overall mortality & $77(38 \%)$ & $122(61 \%)$ & $199(100 \%)$ \\
Mortality (by sex) & $77 / 521(14.8 \%)$ & $122 / 1339(9.1 \%)$ & $199 / 1860(10.7 \%)$ \\
\hline
\end{tabular}




\section{Discussion}

Our findings showed that young men are at higher risk of becoming burn victims in Tehran. Women in Tehran are more at risk of committing suicide by setting themselves on fire. The majority of burns are $>40 \%$ of TBSA with gas and gasoline injury as the most common cause. The demographic and clinical data did not change significantly over the reviewed period. Surgical intervention was the most common treatment procedure, with no significant change in the mortality rate throughout the study. Accidental burns were reported in $85 \%$ of the cases, but $15 \%$ of the burns were suicide-related and mainly among women. In $75 \%$ of suicidal attempts by women, they set themselves on fire.

Our data on self-immolation were mostly in accordance with other studies, which were previously conducted in different regions of Iran. In a survey in 2008, Tehran, Iran, $64 \%$ of 374 incidents from suicide attempts were women and the annual incidence of self-immolation reported as $20.1 \%$ for all burn injuries [5]. In another study from a southern province by Mohammadi et al., suicidal attempts by burning accounted for $21.9 \%$ (284) [6]. Most of these patients (68.2\%) were women and the overall mortality rate in that study was $60.4 \%$. Shakeri et al. performed a survey in a western province of Iran (Kermanshah) [7] where $81.5 \%$ of patients who were admitted because of suicidal self-immolation were women and almost all of them were married, unemployed, and had a low educational level. Of this group, $57.1 \%$ of incidents were precipitated by marital or familial conflicts. The authors concluded that being a married woman is a risk factor for committing self-immolation. The incidence of self-immolation seems to have been an increasing trend during the past decade (of more than 5 times), especially in the north and northwest of Iran [8].

\section{Risk factors}

Young adults were at highest risk of burns in Iran (9). The age groups with the greatest number of burn patients in this study were $21-30$ years (33.9\%) and 31-40 years (20.4\%) old. This finding matches data from developing countries and differs from data in developed countries, where the largest age group is children aged 2-5 years [3,11]. Males accounted for $72 \%$ of patients, which corresponds with most reported studies [9, 12, 13], although some controversial findings have been published elsewhere
$[11,14,15]$. Overall, $15 \%$ of cases of all burns were suicide-related and in $75 \%$ of cases women set themselves on fire. We therefore conclude that being male is a risk factor for committing suicide in Iran. Several studies indicate that women who commit suicide in Iran come from rural areas with a low socio-economic status [16, 17]. Because we have not collected residential data (urban, sub-urban, or rural) in this study, we cannot provide relevant information in this regard.

\section{Settings}

Recent studies have reported a tendency towards increased frequency of self-inflicted burns among men and women [16] with mortality rates of $18 \%-84 \%$ $[6-8,18,19]$. We observed a similar trend in our study. Most burn occurred from natural gas, gasoline and kerosene (51.7\%), followed by open fire (10.2\%). In this regard, our findings were not consistent with those of other studies, which identified open fire as the main cause $[1,3,13,15]$. This discrepancy could be partly explained by the nature of reported burn differences, such as use of unsafe equipment in the workplace or at home, mishandling of gasoline or kerosene in gas stations, and use of kerosene as a main source for home heat by low income families. In addition, 15\% of the burns were suicide-related injuries, in which $75 \%$ of women who committed suicide set themselves on fire in an attempt to end their lives. However, further research is needed to investigate the correlation of source and nature of burns and study the dynamics of suicide causes in Iran and to deliver specific recommendations for identifying the risk factors and prevention.

\section{Prognostic factors}

The overall mortality rate was $10.7 \%$. Our results are consistent with others in demonstrating two main negative prognostic factors: high percentage of involved body surface area [13-15, 19], and younger age [1].

\section{Limitations}

This study is retrospective. Our inability to show a connection between surgical intervention and outcome is probably because of the short period reviewed; a longer review would indeed show that an increase in surgical procedures improves outcome. To fully assess the contribution of surgical management outcome, more specific data are needed 
(e.g., including timing of surgery, graft take rates). Furthermore, the data collected in this study were obtained from only one burn center in Tehran; there are no data regarding nonhospitalized burn patients and burn victims who arrived at other hospitals.

\section{Conclusions}

We sought to identify risk factors and risk groups in order to establish a prevention plan to prevent burns and resulting morbidity and mortality. We found that the groups at highest risk were young adults aged 2130 years and males. Women are at highest risk for suicide attempts with fire. Further study in efforts to reduce risk in these populations are warranted. The safe handling of natural gas, gasoline, and kerosene should be addressed. Early recognition of stress and other factors leading to suicide or suicide attempts in women require urgent further study and identification of causes such that intervention can be implemented by educational programs in schools, community clinics, traditional community centers (e.g., mosques), obstetrics departments, and among religious and social workers is highly desirable. Updated realistic burn management guidelines should be published and disseminated periodically. This kind of database can provide authorities with an up-to-date status of burns and could be a base for focused preventive measures that should be modified periodically. Furthermore, such data will enable us to assess the quality of services provided in different medical centers, with subsequent improvements in treatment delivery and burn management.

The authors have no conflicts of interest to report.

\section{References}

1. Dissanaike S, Rahimi M. Epidemiology of burn injuries: highlighting cultural and socio-demographic aspects. Int Rev Psychiatry. 2009; 21:505-11.

2. Oncul O, Ulkur E, Acar A, Turhan V, Yeniz E, Karacaer Z, Yildiz F. Prospective analysis of nosocomial infections in a burn care unit, Turkey. Indian J Med Res. 2009; 130:758-64.

3. Peck MD. Epidemiology of burns throughout the world. Part I: Distribution and risk factors. Burns. 2011; 37:1087-100.

4. Naghavi M, Abolhassani F, Pourmalek F, Lakeh M, Jafari N, Vaseghi S, et al. The burden of disease and injury in Iran. Popul Health Metr. 2003; 7:9.

5. Taghaddosinejad F, Sheikhazadi A, Behnoush B, Reshadati J, Sabery SH. A survey of suicide by burning in Tehran, Iran. Acta Med Iran. 2010; 48:266-72.
6. Mohammadi AA, Danesh N, Sabet B, Jalaeian H, Mohammadi MK. Self-burning: a common and tragic way of suicide in Fars Province, Iran. IJMS. 2008; $33: 2$.

7. Shakeri J, Tatari F, Sadeghi K, Mohamadi E, Valinia K. Suicide by self-Immolation, a cross sectional study in Kermanshah-Iran.

8. Dastgiri S, Kalankesh LR, Pourafkary N. Epidemiology of self-immolation in the north-west of Iran. Eur J Gen Med. 2005; 2:14-9.

9. Mohammadi-Barzelighi H, Alaghehbandan R, Motevallian A, Alinejad F, Soleimanzadeh-Moghadam S, Sattari M, et al. Epidemiology of severe burn injuries in a Tertiary Burn Centre in Tehran, Iran. Ann Burns Fire Disasters. 2011; 30:59-62.

10. Sh Firoozbakhsh, S Seifirad, V Yamin. Epidemiology of gasoline burn injuries in Tehran, Iran. Iran Red Crescent Med J. 2011; 13:213-4.

11. Khan AA, Rawlins J, Shenton AF, Sharpe DT. The Bradford burn study: the epidemiology of burns presenting to an inner city emergency department. Emerg Med J. 2007; 24:564-6.

12. Dale EL, Mueller MA, Wang L, Fogerty MD, Guy JS, Nthumba PM. Epidemiology of operative burns at Kijabe Hospital from 2006 to 2010: pilot study of a web-based tool for creation of the Kenya Burn Repository. Burns. 2012; 39:788-95.

13. Iqbal T, Saaiq M, Ali Z. Epidemiology and outcome of burns: early experience at the country's first national burns Centre. Burns. 2012; 39:358-62.

14. Othman N, Kendrick1 D. Epidemiology of burn injuries in the East Mediterranean Region: a systematic review. BMC Public Health. 2010; 10: 83.

15. Shanmugakrishnan R, Narayanan V, Thirumalaikolundusubramanian P. Epidemiology of burns in a teaching hospital in south India. Indian $\mathrm{J}$ Plast Surg. 2008; 41:34-7.

16. Suhrabi Z, Delpisheh A, Taghinejad H. Tragedy of women's self-immolation in Iran and developing communities: a review. Int J Burns Trauma. 2012; 2: 93-104.

17. Mosavi F, Shahmohammadi D, Kaffashi A. Epidemiology of suicide in rural area [in Farsi]. Andesheh va Raftar. 2000; 5:4-9.

18. Thombs BD, Bresnick MG, Magyar-Russell G. Who attempts suicide by burning? an analysis of age patterns of mortality by self-inflicted burning in the United States, General Hospital. Psychiatry. 2005; 29: 244-50.

19. Greenbaum AR, Donne J, Wilson D, Dunn KW. Intentional burn injury: an evidence-based, clinical and forensic review. Burns. 2004; 30:628-42. 